\title{
UNDERGRADUATE CONVEXITY
}

From Fourier and Motzkin to Kuhn and Tucker 
This page intentionally left blank 


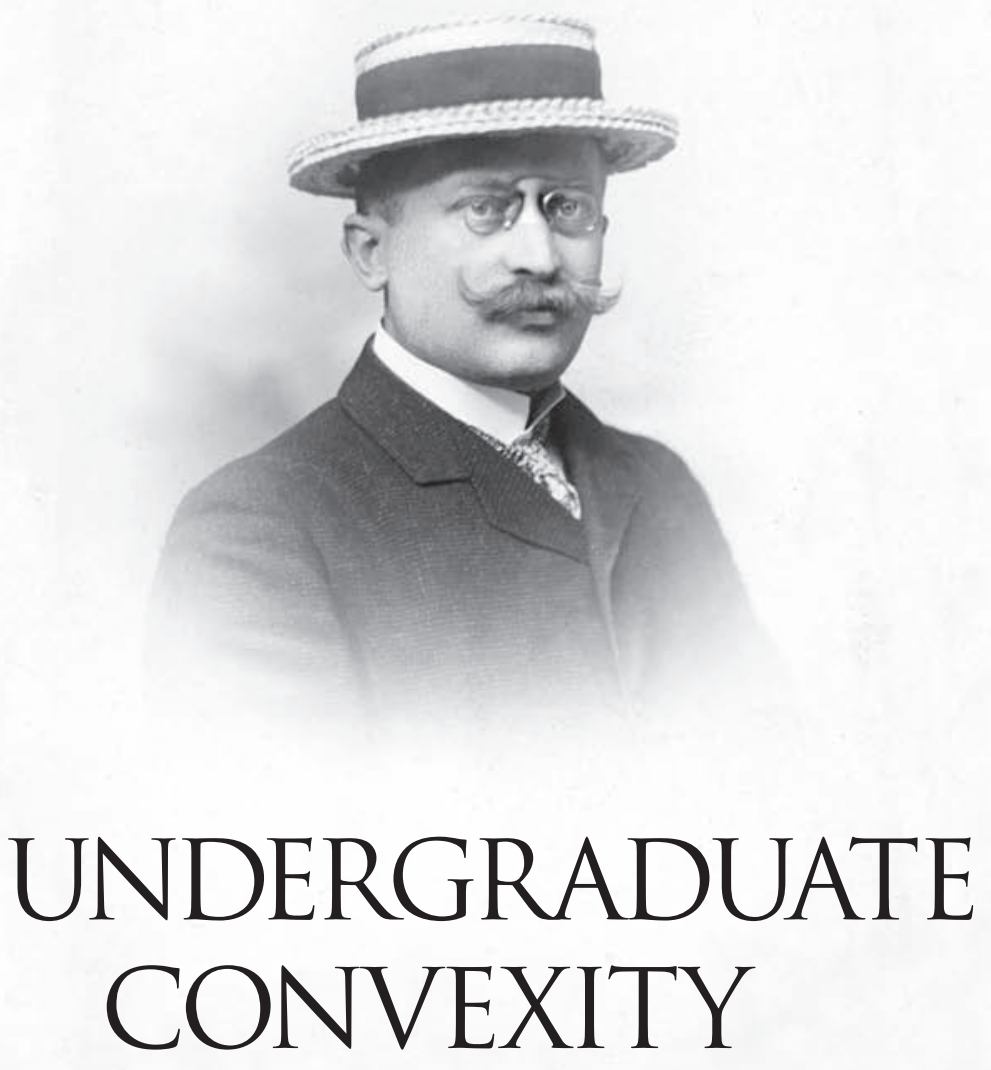

From Fourier and Motzkin to Kuhn and Tucker

Niels Lauritzen

Aarhus University, Denmark 
Published by

World Scientific Publishing Co. Pte. Ltd.

5 Toh Tuck Link, Singapore 596224

USA office: 27 Warren Street, Suite 401-402, Hackensack, NJ 07601

UK office: 57 Shelton Street, Covent Garden, London WC2H 9HE

\section{British Library Cataloguing-in-Publication Data}

A catalogue record for this book is available from the British Library.

Cover image: Johan Ludvig William Valdemar Jensen (1859-1925).

Mathematician and telephone engineer.

Photograph by Vilhelm Rieger (courtesy of the Royal Library, Copenhagen).

\section{UNDERGRADUATE CONVEXITY \\ From Fourier and Motzkin to Kuhn and Tucker}

Copyright (@) 2013 by World Scientific Publishing Co. Pte. Ltd.

All rights reserved. This book, or parts thereof, may not be reproduced in any form or by any means, electronic or mechanical, including photocopying, recording or any information storage and retrieval system now known or to be invented, without written permission from the Publisher.

For photocopying of material in this volume, please pay a copying fee through the Copyright Clearance Center, Inc., 222 Rosewood Drive, Danvers, MA 01923, USA. In this case permission to photocopy is not required from the publisher.

ISBN 978-981-4412-51-3

ISBN 978-981-4452-76-2 (pbk)

Printed in Singapore. 


\section{Preface}

Convexity is a key concept in modern mathematics with rich applications in economics and optimization.

This book is a basic introduction to convexity based on several years of teaching the one-quarter courses Konvekse Mcengder (convex sets) and Konvekse Funktioner (convex functions) to undergraduate students in mathematics, economics and computer science at Aarhus University. The prerequisites are minimal consisting only of first year courses in calculus and linear algebra.

I have attempted to strike a balance between different approaches to convexity in applied and pure mathematics. Compared to the former the mathematics takes a front seat. Compared to some of the latter, a key point is that the ability to carry out computations is considered paramount and a crucial stepping stone to the understanding of abstract concepts e.g., the definition of a face of a convex set does not make much sense before it is viewed in the context of several simple examples and computations.

Chapters 1-6 treat convex subsets from the basics of linear inequalities to Minkowski's theorem on separation of disjoint convex subsets by hyperplanes. The basic idea has been to emphasize part of the rich "finite" theory of polyhedra before entering into the "infinite" theory of closed convex subsets.

Fourier-Motzkin elimination is to linear inequalities what Gaussian elimination is to linear equations. It seems appropriate to begin a course on convexity by introducing this simple, yet powerful method. The prerequisites are barely present. Still the first chapter contains substantial results such as a simple algorithm for linear optimization and the fundamental theorem that projections of polyhedra are themselves polyhedra. 
Before introducing closed convex subsets, several basic definitions and highlights from the polyhedral world are given: a concise treatment of affine subspaces, faces of convex subsets, Bland's rule from the simplex algorithm as a tool for computing with the convex hull, faces of polyhedra, Farkas's lemma, steady states for Markov chains, duality in linear programming, doubly stochastic matrices and the Birkhoff polytope.

The chapter Computations with polyhedra contains a treatment of two important polyhedral algorithms: the double description method and the simplex algorithm. The double description method is related to FourierMotzkin elimination. It is very easily explained in an undergraduate context especially as a vehicle for computing the bounding half spaces of a convex hull.

The simplex algorithm solves linear optimization problems and is somewhat mysterious from a mathematical perspective. There is no obvious reason it should work well. In fact, the famous mathematician John von Neumann never really believed it would perform in practice. The inventor George B. Dantzig also searched for alternate methods for years before confronting experimental data from some of the world's first computers: the simplex algorithm performed amazingly well in practice. Only recently has a mathematical explanation for this phenomenon been given by Spielman and Teng. Our treatment of the simplex algorithm and the simplex tableau deviates from the standard form and works with the polyhedron in its defining space.

The transition to the "continuous" theory of non-polyhedral convex subsets comes after the first five chapters. Here it is proved that closed convex subsets serve as generalizations of polyhedra, since they coincide with arbitrary intersections of affine half spaces. The existence of a supporting hyperplane at a boundary point of a convex subset is proved and Minkowski's theorems on compact convex subsets and separation of disjoint convex subsets are given.

Chapters 7-10 treat convex functions from the basic theory of convex functions of one variable with Jensen's inequality to the Karush-KuhnTucker conditions, dual optimization problems and an outline of an interior point algorithm for solving convex optimization problems in several variables. The setting is almost always the simplest. Great generality is fine when you have lived with a subject for years, but in an introductory course it tends to become a burden. You accomplish less by including more.

The main emphasis is on differentiable convex functions. Since undergraduate knowledge of differentiability may vary, we give an almost com- 
plete review of the theory of differentiability in one and several variables. The only "general" result on convex functions not assuming differentiability is the existence of the subgradient at a point.

An understanding of convex functions of several variables is impossible without knowledge of the finer points of linear algebra over the real numbers. Introducing convex functions of several variables, we also give a thorough review of positive semidefinite matrices and reduction of symmetric matrices. This important part of linear algebra is rarely fully understood at an undergraduate level.

The final chapter treats Convex optimization. The key elements are the Karush-Kuhn-Tucker conditions, how saddle points of the Lagrangian lead to a dual optimization problem and finally an outline of an interior point algorithm using bisection and the modified Newton method. Monographs have been written on these three topics. We only give a brief but selfcontained introduction with simple examples.

\section{Suggestions for teaching a one-semester course}

The amount of material included in this book exceeds a realistic plan for a one-semester undergraduate course on convexity. I consider FourierMotzkin elimination (Chapter 1), affine subspaces (Chapter 2), basics of convex subsets (Chapter 3), the foundational material on polyhedra in Chapter 4, a taste of one of the two algorithms in Chapter 5 and closed convex subsets (Chapter 6) as minimum along with almost all of the material in Chapters 7-10.

The progression of learning depends on the proficiency in linear algebra and calculus. The necessary basic concepts from analysis are introduced in Appendix A. In Appendix B there is a review of linear algebra from the point of view of linear equations leading to the rank of a matrix.

In my view, a too rigid focus on the abstract mathematical details before telling about examples and computations is a major setback in the teaching of mathematics at all levels. Certainly the material in this book benefits from being presented in a computational context with lots of examples.

Aarhus, December 2012 
This page intentionally left blank 


\section{Acknowledgments}

I am extremely grateful to Tage Bai Andersen and Jesper Funch Thomsen for very useful and detailed comments on a second draft for this book. Comments from Kent Andersen, Jens Carsten Jantzen, Anders Nedergaard Jensen and Markus Kiderlen also led to several improvements.

I am an algebraist by training and encountered convexity because of an interest in computational algebra (and computers!). As such, I have benefited immensely over the years from insightful explanations from the following more knowledgeable people: Tage Bai Andersen, Kent Andersen, Kristoffer Arnsfelt Hansen, Peter Bro Miltersen, Marcel Bökstedt, Komei Fukuda, Anders Nedergaard Jensen, Herbert Scarf, Jacob Schach Møller, Andrew du Plessis, Henrik Stetkær, Bernd Sturmfels, Rekha Thomas, Jørgen Tornehave, Jørgen Vesterstrøm and Bent Ørsted.

I am grateful to Jens Carsten Jantzen, Jesper Lützen and Tage Gutmann Madsen for help in tracking down the venerable Jensen inequality postage stamp used for several years by the Department of Mathematical Sciences at University of Copenhagen. Also, thanks to Tinne Hoff Kjeldsen for sharing her expertise on the fascinating history of convexity and optimization.

A very special thanks to the teaching assistants on Konvekse Mcengder and Konvekse Funktioner: Lisbeth Laursen, Jonas Andersen Seebach, Morten Leander Petersen, Rolf Wognsen, Linnea Jørgensen and Dan Zhang. They pointed out several inaccuracies in my lecture notes along the way.

I am grateful to Kwong Lai Fun and Lakshmi Narayanan of World Scientific for their skilled help in the production of this book.

Lars 'daleif' Madsen has been crucial in the technical typesetting with his vast knowledge of $\mathrm{L}_{\mathrm{T}} \mathrm{EX}$ and his usual careful attention to detail.

Finally, Helle and William deserve an abundance of gratitude for their patience and genuine love. 
This page intentionally left blank 


\section{Contents}

Preface $\quad$ v

Acknowledgments $\quad$ ix

1. Fourier-Motzkin elimination 1

1.1 Linear inequalities . . . . . . . . . . . . . . . . 3

1.2 Linear optimization using elimination . . . . . . . . . 8

1.3 Polyhedra . . . . . . . . . . . . . . . . . 9

1.4 Exercises . . . . . . . . . . . . . . . . 13

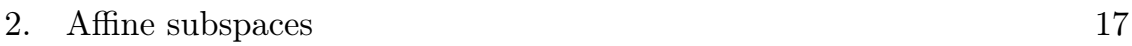

2.1 Definition and basics . . . . . . . . . . . . . 18

2.2 The affine hull . . . . . . . . . . . . . . 20

2.3 Affine subspaces and subspaces . . . . . . . . . . . 21

2.4 Affine independence and the dimension of a subset . . . . 22

2.5 Exercises ....................... 23

3. Convex subsets 27

$3.1 \quad$ Basics . . . . . . . . . . . . . . . . . . . . 28

3.2 The convex hull . . . . . . . . . . . . . 30

3.3 Faces of convex subsets . . . . . . . . . . . . . . . 32

3.4 Convex cones . . . . . . . . . . . . . . . . 36

3.5 Carathéodory's theorem . . . . . . . . . . . . . 41

3.6 The convex hull, simplicial subsets and Bland's rule . . . 45

3.7 Exercises . . . . . . . . . . . . . . . . . . . 48 
4. Polyhedra $\quad 55$

4.1 Faces of polyhedra . . . . . . . . . . . . . . 56

4.2 Extreme points and linear optimization . . . . . . . . 61

4.3 Weyl's theorem .................. 63

4.4 Farkas's lemma .. . . . . . . . . . . . . . . . . 64

4.5 Three applications of Farkas's lemma . . . . . . . . . . 66

4.5.1 Markov chains and steady states . . . . . . . . 66

4.5.2 Gordan's theorem . . . . . . . . . . . . . . . 69

4.5.3 Duality in linear programming . . . . . . . . . . 70

4.6 Minkowski's theorem ................... 74

4.7 Parametrization of polyhedra . . . . . . . . . . . 75

4.8 Doubly stochastic matrices: The Birkhoff polytope . . . . 76

4.8.1 Perfect pairings and doubly stochastic matrices . . 78

4.9 Exercises .................. . . 82

5. Computations with polyhedra 85

5.1 Extreme rays and minimal generators in convex cones . . 86

5.2 Minimal generators of a polyhedral cone . . . . . . . . . 87

5.3 The double description method . . . . . . . . . . . . 90

5.3.1 Converting from half space to vertex representation 95

5.3.2 Converting from vertex to half space representation 96

5.3.3 Computing the convex hull . . . . . . . . . . . 98

5.4 Linear programming and the simplex algorithm . . . . . . 100

5.4.1 Two examples of linear programs . . . . . . . . 102

5.4.2 The simplex algorithm in a special case . . . . . 105

5.4.3 The simplex algorithm for polyhedra in general form 109

5.4.4 The simplicial hack . . . . . . . . . . . . . . . 111

5.4.5 The computational miracle of the simplex tableau . 113

5.4.6 Computing a vertex in a polyhedron . . . . . . . 119

5.5 Exercises ................... 120

6. Closed convex subsets and separating hyperplanes 125

6.1 Closed convex subsets . . . . . . . . . . . . . . 126

6.2 Supporting hyperplanes . . . . . . . . . . . . . . 129

6.3 Separation by hyperplanes . . . . . . . . . . . . . 133

6.4 Exercises .................... . 135

7. Convex functions 141

7.1 Basics .................... 143 
7.2 Jensen's inequality . . . . . . . . . . . . . . . . 145

7.3 Minima of convex functions . . . . . . . . . . . . . 147

7.4 Convex functions of one variable . . . . . . . . . . . 148

7.5 Differentiable functions of one variable . . . . . . . . 150

7.5.1 The Newton-Raphson method for finding roots . . 152

7.5.2 Critical points and extrema . . . . . . . . . 153

7.6 Taylor polynomials . . . . . . . . . . . . 156

7.7 Differentiable convex functions . . . . . . . . . . . . . 159

7.8 Exercises . . . . . . . . . . . . . . . . . . 161

8. Differentiable functions of several variables $\quad 167$

8.1 Differentiability . . . . . . . . . . . . . . 167

8.1.1 The Newton-Raphson method for several variables 171

8.1.2 Local extrema for functions of several variables . . 172

8.2 The chain rule . . . . . . . . . . . . . . . . . . 174

8.3 Lagrange multipliers . . . . . . . . . . . . . . . 176

8.4 The arithmetic-geometric inequality revisited . . . . . 183

8.5 Exercises . . . . . . . . . . . . . . . . . . 184

9. Convex functions of several variables 187

9.1 Subgradients . . . . . . . . . . . . . . . . . . . 188

9.2 Convexity and the Hessian . . . . . . . . . . . . . . 190

9.3 Positive definite and positive semidefinite matrices . . . . 193

9.4 Principal minors and definite matrices . . . . . . . . . 196

9.5 The positive semidefinite cone . . . . . . . . . . . 198

9.6 Reduction of symmetric matrices . . . . . . . . . . . . 201

9.7 The spectral theorem . . . . . . . . . . . . . 205

9.8 Quadratic forms . . . . . . . . . . . . . 208

9.9 Exercises . . . . . . . . . . . . . . . . . . . 214

10. Convex optimization 223

10.1 A geometric optimality criterion . . . . . . . . . . . . 224

10.2 The Karush-Kuhn-Tucker conditions . . . . . . . . . . . 226

10.3 An example . . . . . . . . . . . . . . . . . 230

10.4 The Langrangian, saddle points, duality and game theory 232

10.5 An interior point method . . . . . . . . . . 236

10.5.1 Newtonian descent, exact line search and bisection 238

10.5.2 Polyhedral constraints . . . . . . . . . . . . 240

10.6 Maximizing convex functions over polytopes . . . . . . . . 243 
10.6.1 Convex functions are continuous on open subsets . 244

10.7 Exercises . . . . . . . . . . . . . . . 245

$\begin{array}{lll}\text { Appendix A Analysis } & 253\end{array}$

A.1 Measuring distances . . . . . . . . . . . . . 253

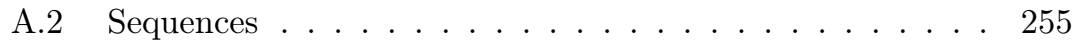

A.2.1 Supremum and infimum . . . . . . . . . 258

A.3 Bounded sequences . . . . . . . . . . . . . . 258

A.4 Closed subsets and open subsets . . . . . . . . . . 259

A.5 The interior and boundary of a set . . . . . . . . 260

A.6 Continuous functions . . . . . . . . . . . . . . . . . . 261

A.7 The main theorem . . . . . . . . . . . . . . 262

A.8 Exercises . . . . . . . . . . . . . . . 262

Appendix B Linear (in)dependence and the rank of a matrix $\quad 265$

B.1 Linear dependence and linear equations . . . . . . . . . 265

B.2 The rank of a matrix . . . . . . . . . . . . . . . 267

B.3 Exercises . . . . . . . . . . . . . . 270

$\begin{array}{ll}\text { Bibliography } & 273\end{array}$

$\begin{array}{ll}\text { Index } & 277\end{array}$ 